AMERICAN JOURNAL OF SCIENTIFIC AND INDUSTRIAL RESEARCH

(C) 2011, Science Hu $\beta$, http://www.scihub.org/AJSIR

ISSN: 2153-649X doi:10.5251/ajsir.2011.2.5.748.754

\title{
A single area load frequency control: a comparative study based on pi, optimal and fuzzy logic controllers.
}

\author{
Ndubisi Samuel .N. \\ Department of Electrical and Electronic Engineering, \\ Enugu State University of Science and Technology, P.M.B. 01660 Enugu Nigeria
}

\begin{abstract}
Load frequency control (LFC) in power systems operation has been a major issue of concern to power system operators. This paper considers design and simulation of a fuzzy logic controller for a single area load frequency control of an isolated power system. The performance of the controller is compared to other existing controllers such as proportional integral (PI) and optimal controllers employed for LFC controls. The result showed an improved frequency and power response during load changes.
\end{abstract}

Keywords: Fuzzy controller, Adaptive controller, Neural network, Control area, Frequency deviation, Nonlinearity

\section{INTRODUCTION}

Isolated power generating units operating in our residential and commercial areas sometimes witness instability due to load variations. The consequence of the above will be a corresponding perturbation in the frequency of supply. Many classical approaches have been used to provide supplementary control which will drag the frequency to normal operating value within a very short time. Such methods include use of proportional integral controllers [1 - 3] applied to act on the load reference setting to change the speed set point. This problem is generally referred to as load frequency control (LFC). The purpose of the integral action is to increase the system type by 1 which will force the final frequency deviation to zero. The control is dependent on the governor speed regulation $\mathrm{R}$ and the integral controller gain $\mathrm{KI}$. This control technique is found to be easy in implementation but generally gives large frequency deviations. Optimal control techniques based on feedback controllers have been proposed to achieve better performance [ $4-6$ ] but it was found that some information about the states are difficult to be known completely. Other approaches based on adaptive neural networks [7 - 8] has been employed to achieve better dynamic responses but the large amount of data and time needed for the training brought some setbacks. Already, some works has been carried out on power systems LFC using fuzzy logic. Higu et al [9] proposed use of frequency deviation and change of frequency deviation as inputs to fuzzy controller while Indulka et.al used area control error (ACE) and change in ACE as inputs to the fuzzy controller [10].

This work proposes a combination of fuzzy logic controller and classical PI controller for effective and efficient control of frequency during load perturbations. The result shows an improved dynamic response even when system nonlinearities are considered.

\section{THE SINGLE AREA MODEL}

Figure 1 below shows the block diagram of an uncontrolled isolated power system. The state variable model for the system is

$$
\dot{X}(t)=A x(t)+B u(t)+L d(t)
$$

Where $A$ is system matrix, $B$ is input distribution matrix, $L$ is disturbance distribution matrix, $X(t)$ is state vector, $u(t)$ is control vector and $d(t)$ is disturbance vector of load changes.

$$
\begin{aligned}
& x(t)=\left[\Delta f, \Delta p_{g}, \Delta p_{v}\right]^{T} \\
& u(t)=\left[\Delta p_{c}\right] \\
& d(t)=\left[\Delta p_{d}\right] \\
& y(t)=\left[\begin{array}{lll}
1 & 0 & 0
\end{array}\right] x, \text { where } y=\Delta f
\end{aligned}
$$


Am. J. Sci. Ind. Res., 2011, 2(5): 748-754

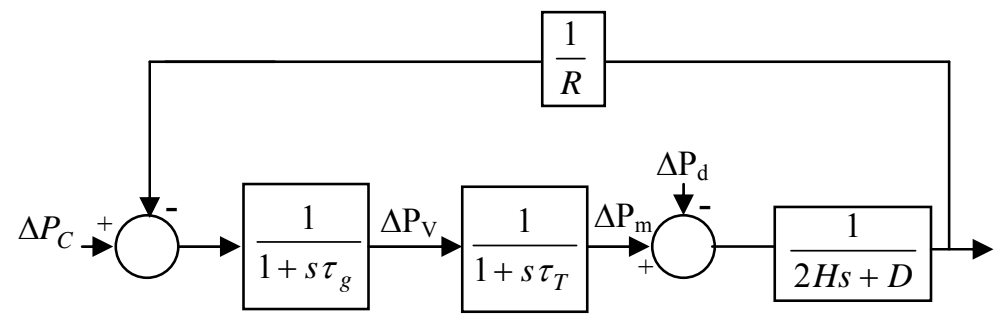

Fig. 1: Model of isolated power system

\section{CONVENTIONAL PI CONTROLLER}

When there is load perturbation in the LFC of figure 1 , it will result to a steady state frequency deviation. The essence of the supplementary control action is to reduce the frequency deviation to zero. The integral controller applied at the load reference setting, changes the speed set point. The controller actually increases the system type by 1 which forces the final frequency deviation to zero (see Fig. 2). The integral controller is adjusted for a satisfactory transient response.

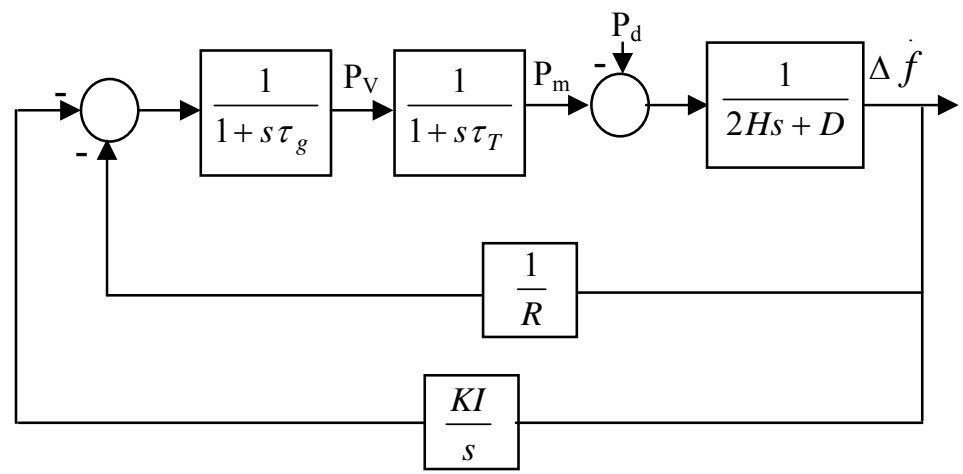

Fig. 2: $A G C$ for isolated power system

\section{OPTIMAL CONTROLLER FOR 1-AREA LFC}

The main goal of optimal regulator or controller design is usually to determine the optimal control law $u^{*}(x, t)$ which can transfer the system from its initial state to final state such that a given performance index is minimized. The applied performance index widely used in optimal control design is the quadratic performance index which is based on minimum-error and minimum - energy critical [].

Let the single area power system be described by

$$
\dot{X}(t)=A x(t)+B u(t)
$$

The problem is to find the vector $k(t)$ of the control law.

$$
u(t)=A x(t)+B u(t)
$$

which minimizes the value of a quadratic performance index $\mathrm{J}$ of the form.

$$
J=\int_{t_{0}}^{t_{f}}\left(x^{\prime} Q x+u^{\prime} R u\right) d t
$$

Subject to equation (2).

$Q=$ positive semidefinite matrix

$R=$ real symmetric matrix.

Using the lagrangian multiplier $(\lambda)$ in optimization, equation (2) and (4) are augmented and the problem reduces to the minimization of the following unconstrained function.

$$
L(x, \lambda, u, t)=\left[x^{\prime} Q x+u^{\prime} R u\right]+\lambda^{\prime}[A x+B u-\dot{x}]
$$

The problem results to

$$
\dot{P}(t)=-p(t) A-A^{\prime} p(t)-q+p(t) B R^{-1} B p(t)
$$

generally referred to as riccati equation where $P(t)=$ time varying positive definite matrix.

The single area system of fig. 1 is modeled to 


$$
\begin{aligned}
& {\left[\begin{array}{c}
\Delta \dot{P}_{v} \\
\Delta \dot{P}_{m} \\
\Delta \dot{F}_{v}
\end{array}\right]=\left[\begin{array}{ccc}
-1 / \tau_{g} & 0 & -1 / R \tau_{g} \\
1 / \tau_{g} & -1 / \tau_{g} & 0 \\
0 & 1 / 2 H & -\mathrm{D} / 2 \mathrm{H}
\end{array}\right]} \\
& +\left[\begin{array}{c}
0 \\
0 \\
-1 / 2 H
\end{array}\right] \Delta P_{L}+\left[\begin{array}{c}
1 / \tau_{g} \\
0 \\
0
\end{array}\right] \Delta \mathrm{P}_{\mathrm{ref}}
\end{aligned}
$$

which is of the form $\dot{X}(t)=A x+B u+L d$. The optimal control scheme for a single area is simulated in section 3 of this work.

\section{FUZZY CONTROL FOR A SINGLE AREA OAD FREQUENCY CONTROL (LFC)}

Fuzzy control is based on a logical system called fuzzy logic. It is much close in spirit to human thinking and natural language than classical logical systems. The LFC as has been reported in several papers is to maintain balance between production and consumption of electrical power. Due to the complexity and multi-variable nature of power systems, a

conventional control method has not provided satisfactory solutions. The fuzzy logic control has tried to handle the robustness, reliability and nonlinearities associated with power system controls.

The block diagram of fig. 3 shows the single area fuzzy load frequency controller. It uses two input membership variables (frequency deviation $\Delta f$ and change in frequency deviation $\dot{\Delta} f$ ). Fig. $a, b, c$ shows the membership functions for the $\Delta f, \dot{\Delta} f$ and the control output $u$. The input signals are first expressed in some linguistic variables using fuzzy set notations such as large positive (LP), medium positive (MP) small positive (SP), very small (VS), small negative (SN), medium negative (MN) and large negative (LN). Table 1 shows a set of decision rules, also expressed in linguistic variables relating input signals to the output (control) signal. The rules interpreted as follows:

If $\Delta f$ is LP and $\dot{\Delta} f$ is MP then $\mathrm{u}$ is LP

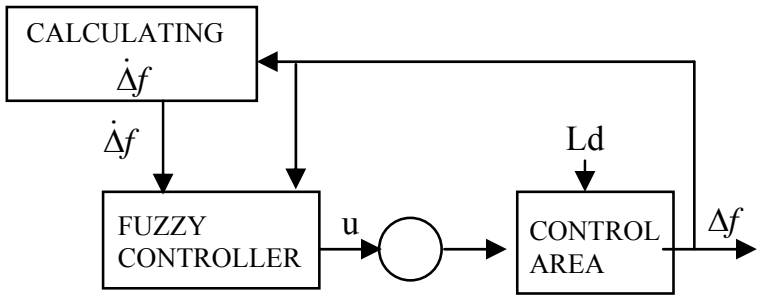

Fig. 3: Single Area Fuzzy

\section{SIMULATIONS AND RESULTS}

The following parameters of an isolated power station are assumed

$$
\tau_{T}=0.5 \mathrm{sec}, \tau_{g}=0.2 \mathrm{sec}, \mathrm{H}=5 \mathrm{sec}, \mathrm{R}
$$

$=0.05$ per unit, $\mathrm{f}=50 \mathrm{~Hz}$.

(a) For a $250 \mathrm{MW}$ turbine rated output and sudden load change of $50 \mathrm{MW}\left(\mathrm{P}_{\mathrm{L}}=0.2\right.$ per unit), the frequency deviation was simulated without any controller and the response is as shown in Fig. 5.

(b) For the same parameters and load change as in (a), but now with an integral controller, simulation was carried out for various values of integral gain $K I$ and result shown in fig. 6

(c) With the same data as in (a) and (b) but with optimal controller employed using riccati equation and with following specifications. Performance index.

$$
I=\int_{0}^{\infty}\left(20 x_{1}^{2}+15 x_{2}^{2}+5 x_{3}^{2}+0.15 u^{2}\right) d t
$$

Weight matrix $Q=\left[\begin{array}{lllllll}20 & 0 & 0,0 & 10 & 0,0 & 0 & 5\end{array}\right]$

$$
R=0.15
$$

The simulation is carried, the optimal gain matrix $K$ is obtained and the frequency deviation response is shown fig. 7. $\left(\mathrm{X}_{1}=\right.$ Governor power deviation $\Delta \mathrm{P}_{\mathrm{V}}$, $\mathrm{X}_{2}=$ turbine power deviation $\Delta \mathrm{P}_{\mathrm{m}}, \mathrm{X}_{3}=$ frequency deviation $\Delta f$ ).

(d) A fuzzy logic PI controller is employed with all the data as before. The simulation is carried out and the frequency deviation step response is as shown in fig. 8 
Am. J. Sci. Ind. Res., 2011, 2(5): 748-754

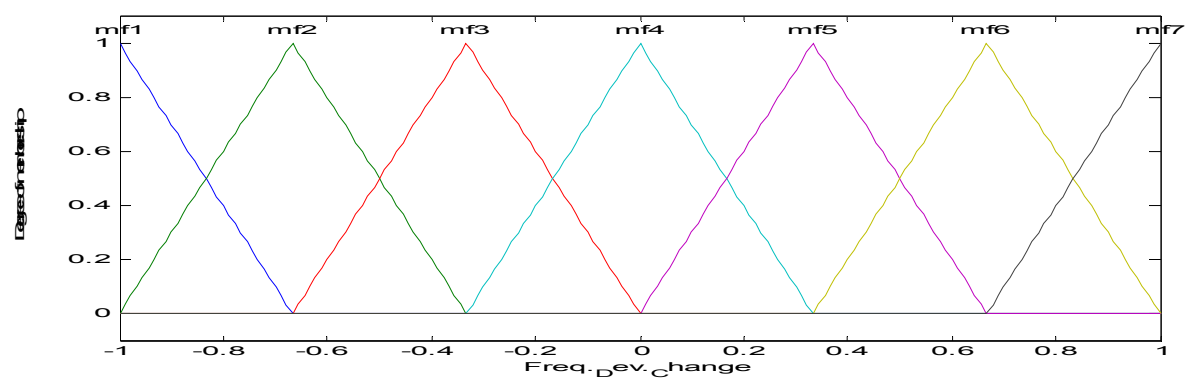

(a) membership function for the error $(\Delta f)$

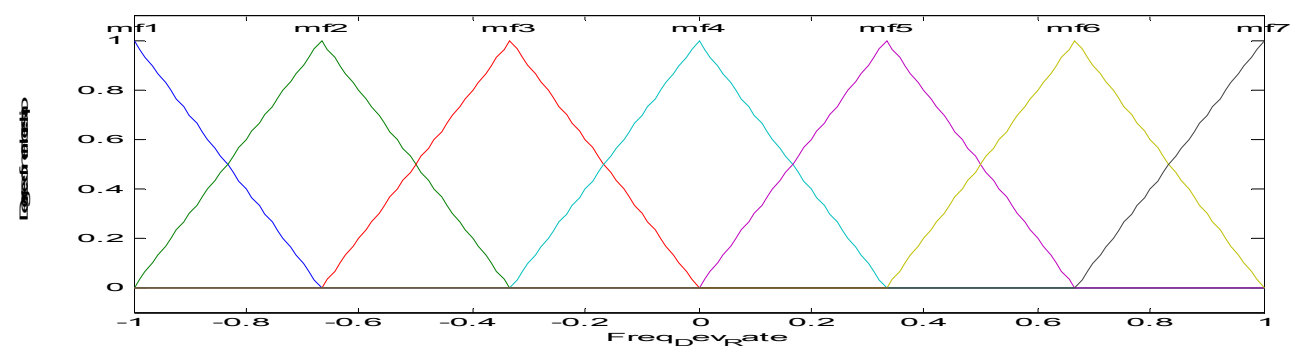

(b) membership function for the change in error $(\Delta f)$

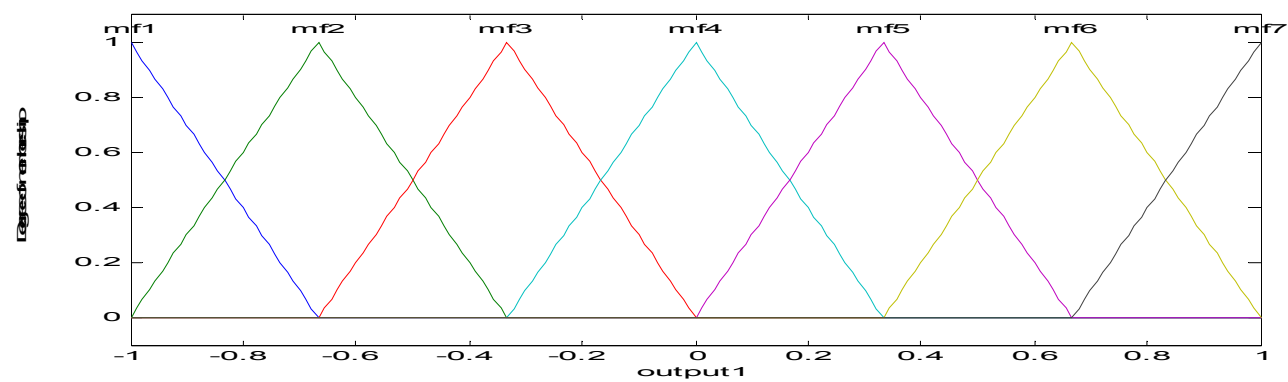

(c) membership function for the output $u$

Fig. $4(a, b, c): \quad$ membership functions for inputs and output

Table 1: Decision Rules

\begin{tabular}{|c|c|c|c|c|c|c|c|c|}
\hline & \multicolumn{7}{|c|}{ Frequency Deviation Rate $\dot{\Delta} f$} \\
\hline & & $\begin{array}{r}1 \\
\text { LN }\end{array}$ & $\begin{array}{c}2 \\
M N\end{array}$ & $\begin{array}{c}3 \\
\text { SN }\end{array}$ & $\begin{array}{c}4 \\
\text { VS }\end{array}$ & $\begin{array}{r}5 \\
\text { SP }\end{array}$ & $\begin{array}{c}6 \\
M P\end{array}$ & $\begin{array}{r}7 \\
\text { LP }\end{array}$ \\
\hline $\begin{array}{c}\text { Frequency } \\
\text { Deviation } \\
\Delta f\end{array}$ & $\begin{array}{l}\text { LP } \\
\text { MP } \\
\text { SP } \\
\text { VS } \\
\text { SN } \\
\text { MN } \\
\text { LN }\end{array}$ & $\begin{array}{l}\text { VS } \\
\text { SN } \\
\text { MN } \\
\text { MN } \\
\text { LN } \\
\text { LN } \\
\text { LN }\end{array}$ & $\begin{array}{l}\text { SP } \\
\text { VS } \\
\text { SN } \\
\text { MN } \\
\text { MN } \\
\text { LN } \\
\text { LN }\end{array}$ & $\begin{array}{l}\text { MP } \\
\text { SP } \\
\text { VS } \\
\text { SN } \\
\text { SN } \\
\text { MN } \\
\text { LN }\end{array}$ & $\begin{array}{l}\text { LP } \\
\text { MP } \\
\text { SP } \\
\text { VS } \\
\text { SN } \\
\text { MN } \\
\text { LN }\end{array}$ & $\begin{array}{l}\text { LP } \\
\text { MP } \\
\text { SP } \\
\text { SP } \\
\text { VS } \\
\text { SN } \\
\text { MN }\end{array}$ & $\begin{array}{l}\text { LP } \\
\text { LP } \\
\text { MP } \\
\text { MP } \\
\text { SP } \\
\text { VS } \\
\text { SN }\end{array}$ & $\begin{array}{l}\text { LP } \\
\text { LP } \\
\text { LP } \\
\text { MP } \\
\text { MP } \\
\text { SP }\end{array}$ \\
\hline
\end{tabular}




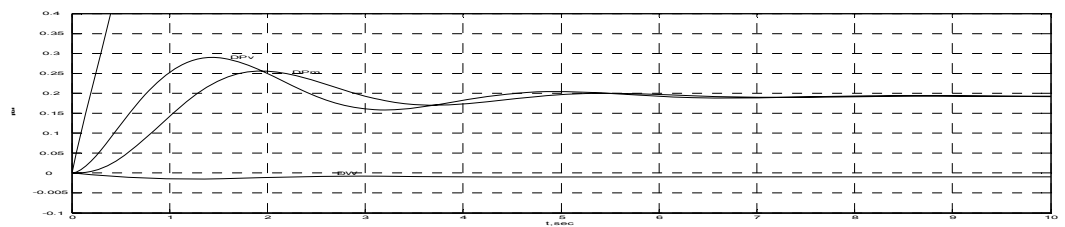

Fig.5 (i) Step response (DPv,DPm,Dw) without supplementary controller

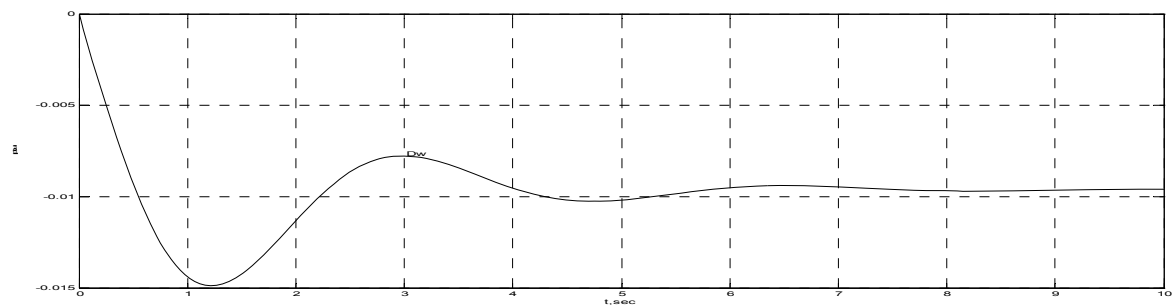

Fig.5(ii):Frequency deviation step response(Dw) without supplementary controller

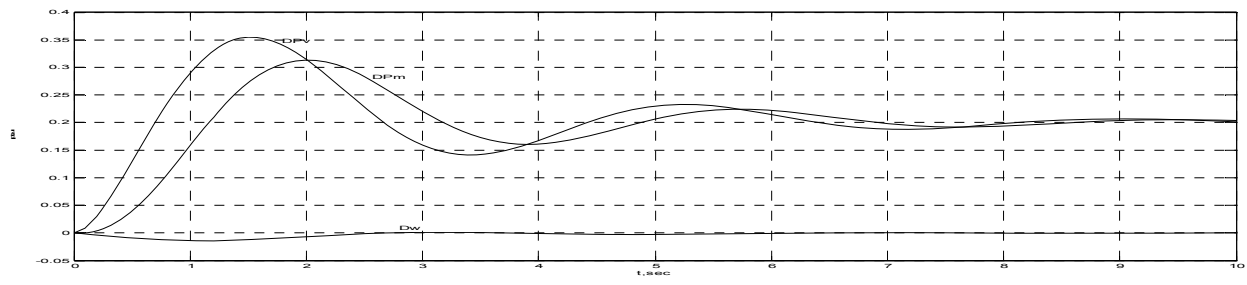

Fig. 6i: Step responses (DPv,DPm,Dw) with conventional PI controller

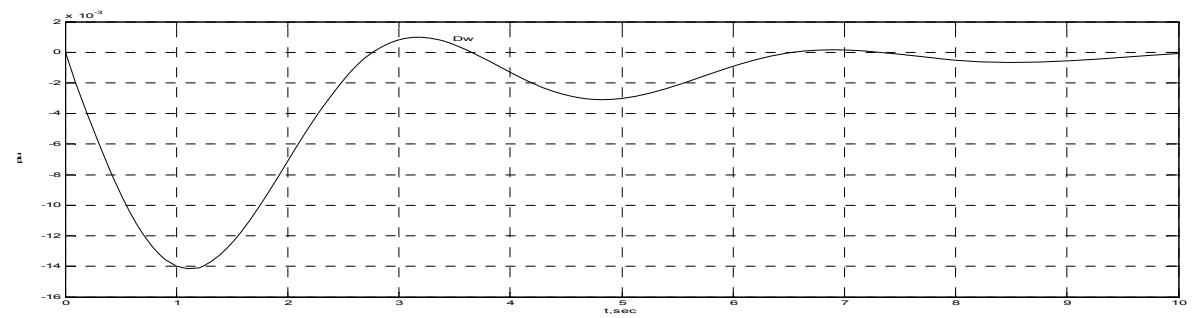

Fig6ii: Frequency deviation step responses (Dw) with conventional PI controller

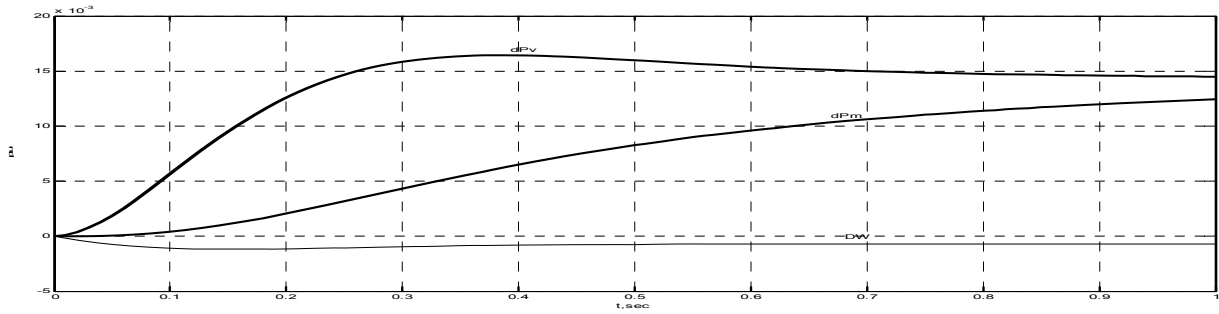

Fig7i: Frequency deviation step responses with LQR controller. 
Am. J. Sci. Ind. Res., 2011, 2(5): 748-754

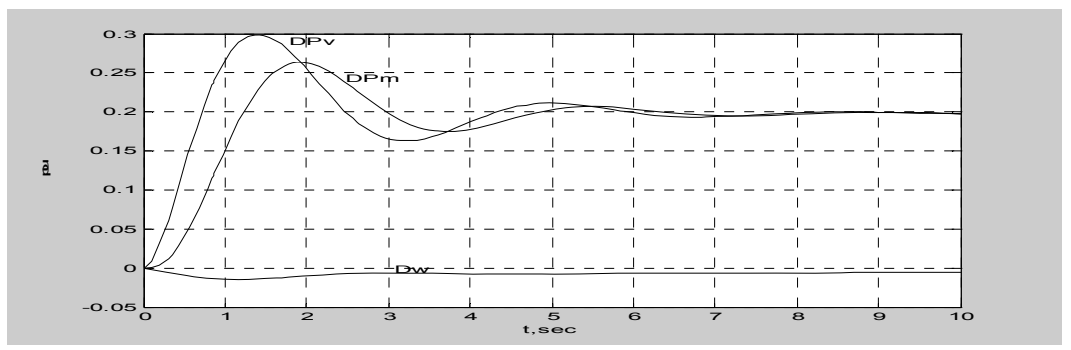

Fig. 8i: Step responses (DPv,DPm,Dw) with fuzzy PI controller.
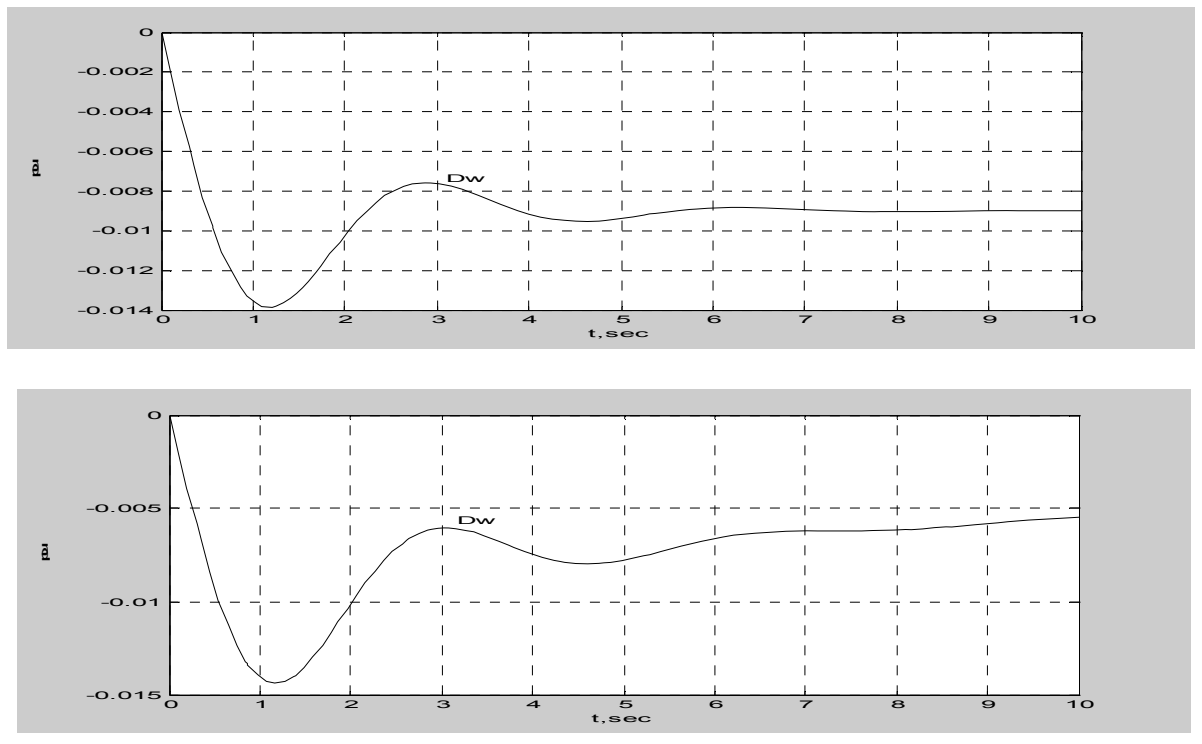

Fig. 8ii: Frequency deviation step responses (Dw) with fuzzy PI controller

\section{CONCLUSION}

A comparative study of performances of proportional integral, optimal and fuzzy logic based controllers to a single area load frequency control scheme is carried out in this work. The PI controller is seen to perform very well as regards to the frequency and power fluctuations during region but may oscillate outside it.

The optimal controller showed good performance but highly depends on the choice of the weighting matrices $Q$ and $R$. Wrong choice of these may result to the frequency and power oscillating during disturbances. Fuzzy logic controller gave a very good stable performance of frequency and power responses during large disturbances but still depends on the expert knowledge in choosing the rule base. A close analysis of the results shows that the fuzzy logic controller performs well even when the disturbance is very large and with non linearity of the power system taken into consideration.

\section{REFERENCES}

[1] Kundur, P., Power System Stability and Control, McGraw - Hill Book Company, New York, 1994.

[2] Saadat, H., Power system Analysis, McGraw - Hill Book Company, New York, 1999.

[3] Aldeen, M. and Marah, J.F., "Decentralized Proportional - Plus-Integral Design Method for Interconnected Power Systems", IEE Proceedings-C, Vol. 138, No. 4, 1991.

[4] Yamashika, K., and Miyagi, H., "Multivariable selfTuning Regulator for Load Frequency Control System with Interaction of Voltage on Load Demand", IEEE Proceedings-D, Vol. 138, NO. 2, March 1999.

[5] Aldeen M., "A Fresh Approach to the LQR Problems with Application to Power Systems", Proc. of Int. Power Engineering Conf., Singapore Vol. 1, 1993, Pp. $374-379$. 
[6] Pan, C. I., and Liaw L.M. "AN Adaptive Controller for Power System Load - Frequency Control", IEEE on Power System. Vol. 4, No. 1, Feb. 1989, Pp 122 128.

[7] Dyukanovic, M., et.al "Two-Area Load Frequency Control with Neural Networks,: Proc. 1993. North American Power Symposium, Pp. 161 - 169.

[8] Brich, A. P, et.al, "Neural Network Assisted Load Frequency Control", $28^{\text {th }}$ University Power Engineering Conf. Proc. Vol. 2, 1993, Pp. 518 - 521.
[9] Hsu, Y., and Cheng, C., "Load Frequency Control using Fuzzy Logic," Int. Conf. on High Technology in the Power Industry, 1991, Pp. $32-38$.

[10] Indulka, C. S., and Raj, B., "Application of Fuzzy Controller to Automatic Generation Control," Electric Machines and Power Systems, Vol. 23, No. 2, MarApr. 1995, pp. 209 - 220.

[11] Fuzzy Logic Toolbox user's Guide. 